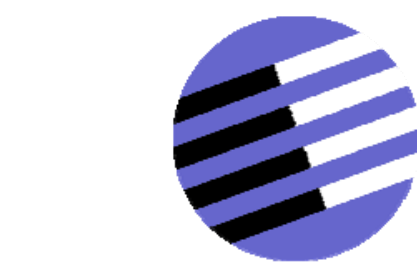

GOVERNANCE AND THE EFFICIENCY

OF ECONOMIC SYSTEMS

GESY

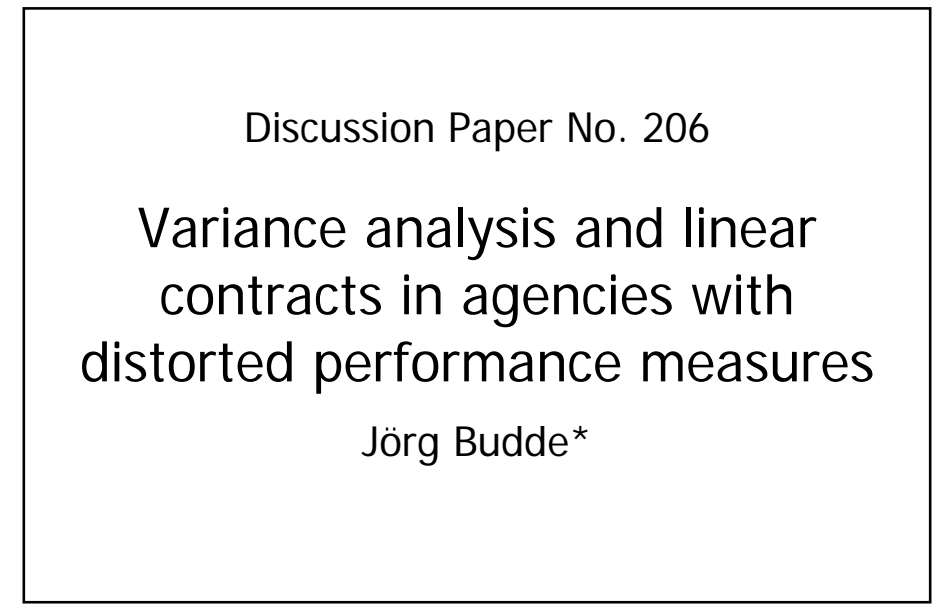

April 2007

*Jörg Budde, Department of Economics, University of Bonn, Adenauerallee 24-42, D-53113 Bonn; phone: +49-228-739247, Joerg.Budde@uni-bonn.de

Financial support from the Deutsche Forschungsgemeinschaft through SFB/TR 15 is gratefully acknowledged. 


\title{
Variance analysis and linear contracts in agencies with distorted performance measures*
}

\author{
Jörg Budde ${ }^{\dagger}$ \\ revised version of Variance analysis, linear contracts and congruity in \\ multi-task agencies, April 2007
}

\footnotetext{
${ }^{*}$ I would like to thank Dominique Demougin, Alfred Luhmer, Christian Lukas, Kai Mitusch, Ulf Schiller, two anonymous referees and conference participants in Athens and Vallendar for helpful comments. Financial support by the Deutsche Forschungsgemeinschaft through SFB/TR 15 is gratefully acknowledged.

${ }^{\dagger}$ Department of Economics, University of Bonn, Adenauerallee 24-42, D-53113 Bonn; phone: +49228-739247; email: Joerg.Budde@uni-bonn.de
} 


\title{
Variance analysis and linear contracts in agencies with distorted performance measures
}

\begin{abstract}
This paper investigates the role of variance analysis procedures in aligning objectives under the condition of distorted performance measurement. A riskneutral agency with linear contracts is analyzed, whereby the agent receives postcontract, pre-decision information on his productivity. If the performance measure is informative with respect to the agent's marginal product concerning the principal's objective, variance investigation can alleviate effort misallocation. These results carry over to a participative budgeting situation, but in this case the variance investigation procedures are less demanding.
\end{abstract}




\section{Introduction}

Variance investigation has frequently been the subject of management accounting research in terms of both facilitating and influencing decision-making. Such research has often concentrated on incentive effects. From the agency perspective, the literature has mainly focused on the trade-off between risk-sharing and incentives. This viewpoint essentially reduces the agency problem to the question of which contractual agreement can induce a certain desired action at minimal cost. Several results on the use of variance analysis procedures have been derived by applying the Holmström (1979) informativeness criterion. The crucial requirement for useful variance investigation in this context is the provision of additional information with respect to the agent's action. If observation of an overall result is not statistically sufficient, there are potential gains from analyzing further details regarding the agent's actions (Baiman and Demski 1980a). ${ }^{1}$

In the last decade, however, economic agency research has emphasized the misallocation of effort rather than the trade-off between risk and incentives as the central issue in the provision of incentives. Starting with Holmström and Milgrom (1991), a rich literature has analyzed the effects of dysfunctional behavior. This problem may arise whenever an agent's performance indicator does not fully accord with his principal's objective, which can occur for a variety of reasons. On the one hand, the principal could have a non-contractible objective such as the value of a privately traded firm. On the other hand, the objective might be a very risky measure of the agent's performance and result in a high risk premium to be paid. In both cases, the principal may seek alternative performance measures to provide contractual incentives. Such measures,

\footnotetext{
${ }^{1}$ In practical applications, these benefits of course have to be weighed against the cost of data gathering, and conditional monitoring might become advantageous. See Baiman and Demski (1980b) and Young (1986) for a detailed analysis.
} 
however, may induce effort allocations that do not coincide with those preferred by the principal, particularly if the agent performs a variety of tasks.

Multi-dimensional effort may result for two reasons. First, the agent might work on different tasks and have to decide not only on the total amount of effort, but also on where to put it. Second, the agent's productivity might depend on some state of nature that he observes before choosing his action, leading to a state-contingent action. If the principal's objective and the performance measure are influenced by the state of nature in different ways, a similar misallocation problem arises.

From an accounting perspective, an obvious question in both cases is whether management accounting procedures such as variance analysis can help to alleviate the problem. To answer this question, I first analyze how additional input information can best be incorporated into a linear contract. Building on these results, their relation to variance analysis procedures is then studied. It emerges that certain special variances can be naturally interpreted as predictors of the agent's impact on the firm's objective. Consequently, they appear in the agent's compensation function. Distortion of performance measurements is therefore another rationale for tying compensation to variances in corporate practice. I derive these results for the second scenario of post-contractual private information, for which the adoption of variance analysis procedures and participative budgeting has a more natural interpretation. However, since the general effects of distortion are the same in both settings, the results on variance investigation apply to the classical multi-task setting as well, with a slightly different interpretation of variances.

In a broader sense, my aim is to connect two branches of literature: one on distortion in performance measurement, and the other on the use of variance analysis procedures for incentive contracting. In the first respect, the paper is most closely related to Baker (1992) and Feltham and Xie (1994). Like Baker, I consider the com- 
bined use of output and input data to improve the congruity of performance measures. In addition, I consider the role of participation in budgeting and discuss the relation of performance to accounting data. In this last respect, the paper is more closely related to the work of Feltham and Xie, but they do not consider the use of accounting procedures such as variance analysis. In this regard, I follow Darrough (1988) and Kloock and Schiller (1997). Kloock and Schiller describe different decomposition methods proposed for variance analysis, particularly in the German cost accounting literature. I refer to them when I describe the optimal contract in terms of variance decomposition results. Kloock and Schiller present only verbal arguments on the use of variance investigation for incentive purposes. I find evidence supporting their statements in a quantitative interpretation of the model. Darrough (1988) considers the use of ex post budgets in splitting the efficiency variance in cost accounting. Although Darrough does not explicitly employ an agency model, both her work and mine use the agent's reaction to his pre-decision information. While in Darrough's paper this information is publicly observable ex post, in this paper only its impact on the performance measure can be used for contracting. Accordingly, in Darrough's paper an agent would always choose the first-best input mix, whereas in my model implementation depends on the relation of the principal's objective and the agent's performance measure.

The remainder of the paper is organized as follows. In Section 2, a general model of distorted performance measurement under private information is described. Section 3 studies the impact of additional information and the role of variance analysis procedures. Section 4 considers participative budgeting, and Section 5 draws conclusions and discusses directions for future research. 


\section{Distorted performance measurement under private information}

To introduce the problem of distorted performance measurement under private information, I adapt a model studied by Baker (1992). For this purpose, consider a riskneutral principal hiring a risk-neutral agent to perform a certain task on his behalf. The agent takes an action $a \in \mathbb{R}^{+}$, which, along with a random variable $\delta \in[\underline{\delta}, \bar{\delta}] \subset \mathbb{R}^{+}$, determines the realization of the principal's objective $V(a, \delta)=\delta a$. By choosing $a$, the agent incurs a private cost $C(a)=a^{2} / 2$. Thus, maximizing the total surplus ${ }^{2}$ $V-C$ would require $a=\delta$, equating the marginal product $\delta$ and the marginal cost $a$ of the agent's effort. To study asymmetric information and performance measure distortion, I assume that $\delta$ cannot be observed by the contracting parties. Only the agent receives a signal $\phi \in \mathbb{R}^{+}$, from which he imperfectly infers the realization of $\delta$. Up to this point, the setting is a linear-quadratic specification of the model studied in the standard agency theory (Harris and Raviv 1979), for which, owing to the agent's risk-neutrality, a first-best solution could be achieved by selling the business to the agent. The agent would use his information to maximize the conditional expectation of $V-C$, choosing an action $a^{F B}=\mathrm{E}[\delta \mid \phi]$.

I rule out this trivial case by assuming that $V$ is not the value of the firm as a whole, but only the agent's contribution to the firm's value, which cannot be separated from the remaining assets and sold to the manager. Instead of selling the firm, incentives therefore have to be provided by tying the agent's compensation to a contractible performance measure $P$. For instance, if $V$ is the value added to a privately traded firm, $P$ might be some measure of short-term success such as profit or ROI.

Since such short-term performance may at least partly determine the total value added by the manager, it is evident that we can allow that $V$ and $P$ are correlated. I

\footnotetext{
${ }^{2} \mathrm{We}$ can focus on the total surplus of the agency because both parties are risk-neutral and no liability constraints are considered.
} 
do so by assuming that $P(a, \phi, \nu)=\phi a+\nu$, where the sensitivity ${ }^{3}$ of the performance measure is the agent's private information $\phi$. The noise term $\nu \in \mathbb{R}$ with $\mathrm{E}[\nu]=0$ is unobservable to both parties, and ensures that a forcing contract cannot be written. Instead, the principal offers a linear payment scheme

$$
S=s_{0}+s P
$$

to the agent. ${ }^{4}$ At the time this contract is signed, neither the principal nor the agent has information about the realization of $\phi$, and they have common beliefs about its distribution. Before the agent chooses his action, however, $\phi$ becomes observable to him. From this post-contractual information asymmetry, a chance to improve the agent's decision, as well as the problem of distorted performance measurement, arises.

To see this, first consider a situation in which the agent does not observe $\phi$. Ignorant of $\phi$, he chooses his action $a^{N I}=s \mathrm{E}[\phi]$ to maximize his expected utility $s_{0}+s \mathrm{E}[P-C]=s_{0}+s \mathrm{E}[\phi] a-a^{2} / 2$, and the principal can induce an action maximizing the expected total surplus $\mathrm{E}[V-C]$ by setting $s^{N I}=\mathrm{E}[\delta] / \mathrm{E}[\phi]$. The agent chooses $a^{N I}=\mathrm{E}[\delta]$, and an expected total surplus of $\mathrm{E}[V-C]^{N I}=\mathrm{E}[\delta]^{2} / 2$ accrues to the agency.

Since the agent observes $\phi$, however, he will choose his action according to the realization of $\phi$ to maximize his expected utility $s_{0}+s \mathrm{E}[P-C \mid \phi]=\phi a-a^{2} / 2$. To account for this, the principal will choose the contract parameters to maximize the expected total surplus

$$
E[V-S \mid s]=\mathrm{E}\left[\delta a(s)-a(s)^{2} / 2\right]=s \mathrm{E}[\delta \phi]-\frac{s^{2}}{2} \mathrm{E}\left[\phi^{2}\right]
$$

\footnotetext{
${ }^{3}$ See Banker and Datar (1989) for a definition.

${ }^{4}$ In general, the principal could make use of the agent's information by offering menus of contracts. These are analyzed in Section 4. For the time being, assume that $\phi$ cannot be communicated, and the same contract has to be offered for all its realizations.
} 
The optimal contract specifies $s^{P I}=\mathrm{E}[\delta \phi] / \mathrm{E}\left[\phi^{2}\right]$ (cf. Baker 1992), leading to a total surplus

$$
\mathrm{E}[V-C]^{P I}=\frac{\mathrm{E}[\delta \phi]^{2}}{2 \mathrm{E}\left[\phi^{2}\right]}
$$

Comparing the two outcomes yields the first result, concerning the value of information:

Proposition 1 In the risk-neutral agency setting with unobservable effort and without communication, the value of information to the agency may be positive or negative.

Proof First, assume $\delta$ and $\phi$ are such that $\mathrm{E}[\delta \phi]=0$. Then $\mathrm{E}[V-S]^{P I}=0 \leq$ $\mathrm{E}[V-S]^{N I}$, and the value of information is negative. On the contrary, if $\phi=\delta$, for example, $\mathrm{E}[V-S]^{P I}=\mathrm{E}\left[\delta^{2}\right] / 2 \geq \mathrm{E}[V-S]^{N I}$, and the value of information is positive.

Proposition 1 is a well-known result in the standard agency model (Demski 1980, p. 97f.; Christensen 1981, p. 669ff.). Here, it is asserted for two reasons. First, it serves as a benchmark for the variance investigation procedures analyzed in the next section. Second, the proof of Proposition 1 shows that if $\phi=\delta$, the full-information outcome can be achieved. This raises the question as to how a weaker relation of the two random variables affects the agency's surplus. Baker (1992) states that it is determined by the correlation of the two variables. Indeed, if (2) is written as

$$
\mathrm{E}[V-C]^{P I}=\frac{(\mathrm{E}[\delta] \mathrm{E}[\phi]+\operatorname{Cov}[\delta, \phi])^{2}}{2 \mathrm{E}\left[\phi^{2}\right]},
$$

it is obvious that the surplus is higher if $\delta$ and $\phi$ show a stronger (positive) correlation. However, an inspection of (3) also makes it clear that the performance measure affects the agency's surplus not only by its correlation to the firm's value, but also by its absolute level. This may be illustrated by the following example. 
Example Let the marginal product $\delta$ be uniformly distributed on the unit interval $[0,1]$ and the performance measure's sensitivity be $\phi=h+\delta$, where $h \in \mathbb{R}^{+}$is a constant known to both contracting parties. Obviously, $\delta$ and $\phi$ are perfectly correlated. The expected total surplus $\mathrm{E}[V-C]^{N I}=(2+3 h)^{2} /\left(12+36 h+36 h^{2}\right)$, however, depends on the value of $h$. It is equal to the full-information solution if $h=0$, and approaches the no-information solution for $h \rightarrow \infty$.

On first glance, the example seems to conflict with Baker's (1992) result that if the marginal products of $V$ and $P$ are perfectly correlated and have the same varianceas is the case in the example-the first-best solution is obtained. The discrepancy arises from the fact that Baker normalizes the performance measure with respect to its marginal product, such that, translated to the present model, $\delta$ and $\phi$ would have the same expectation. Demanding this in the above example, however, would lead to $\delta=\phi$, in which case the first-best solution is obviously obtained.

¿From an accounting perspective, the example may well be interpreted in terms of the controllability principle. Since the agent at least partly controls the variable $P$, he is held responsible for it, especially because he obtains knowledge of uncontrollable effects before be chooses his action. This accounts for a refinement of the controllability principle, stating that managers should be held responsible for those numbers they are supposed to pay attention to (cf. Merchant and Van der Stede 2003, p. 464). Since the principal wishes the agent to account for his private information $\phi$, the performance measure $P$ is of particular value for incentive purposes. The extent to which it is used, however, depends on the impact of $h$, because $h$ is neither controlled by the agent nor is it predictive of the economic outcome $V$. Thus, to focus the agent's attention on the value-relevant parts of his measured performance, the principal would be interested in filtering out $h$. In the next section, variance analysis is introduced as a general device 
for this purpose.

\section{Use of additional input information and variance analysis}

The above example shows that a performance measure may be almost valueless, even if it is perfectly correlated with the principal's objective. The reason for this counterintuitive result is that although the incentive contract could account for any variation in the marginal product $\delta$ (this would be done by fixing $s=1$ ), the principal will not make use of this opportunity because the absolute level of effort would be too high. Consequently, he will choose a lower level of incentives, which obviously will not fully account for the possible variations of $\delta$.

To adjust the absolute level of effort, the contract has to incorporate additional information related to the agent's input $a$ instead of the output number $P$. Such information is frequently considered in variance investigation procedures, which try to explain deviations between budgeted and realized output numbers by incorporating additional input information. In the present setting, the difference $\Delta P=P^{R}-P^{B}$ of the realized value $P^{R}$ and the budgeted amount $P^{B}$ of the performance measure could be split into a component $\Delta^{\phi} P$ due to the variation of $\phi$, and a component $\Delta^{a} P$ due to the deviation of $a$,

$$
\Delta P=P^{R}-P^{B}=\underbrace{\left(P^{R}-\phi^{B} a^{R}\right)}_{\Delta^{\phi} P}+\underbrace{\left(\phi^{B} a^{R}-\phi^{B} a^{B}\right)}_{\Delta^{a} P},
$$

where the superscripts $B$ and $R$ refer to the budgets and realizations, respectively. ${ }^{5}$

To conduct this decomposition, a measure of the agent's input needs to be available. For simplicity, assume that $a$ is observed by the contracting parties and can be used for

\footnotetext{
${ }^{5}$ The realized value of $\phi$ cannot enter the calculation, since $\phi$ cannot be observed by the principal. Instead, it is implicitly inferred from $P$ by the residual deviation $P^{R}-\phi^{B} a^{R}$, which cannot be explained by the deviation of $a$.
} 
performance evaluation. ${ }^{6}$ Under the linearity assumption, the compensation contract becomes

$$
S=s_{0}+s_{1} a+s_{2} P
$$

The agent's action under this contract will be $a\left(s_{1}, s_{2}\right)=s_{1}+s_{2} \phi$, which enables the principal to control the absolute level of effort. In the optimal contract, he will fix ${ }^{7}$

$$
s_{1}=\mathrm{E}[\delta]-s_{2} \mathrm{E}[\phi] \quad \text { and } \quad s_{2}=\frac{\operatorname{Cov}[\delta, \phi]}{\operatorname{Var}[\phi]} .
$$

This allows us to write the agent's compensation in the form

$$
S=s_{0}+\mathrm{E}[\delta] a+\frac{\operatorname{Cov}[\delta, \phi]}{\operatorname{Var}[\phi]}(P-\mathrm{E}[\phi] a) .
$$

Owing to the assumption of a two-piece-rate contract, the optimal compensation can of course be written as a function of some variance. The more interesting question is how (7) relates to the variance decomposition described in (4), and how this procedure corresponds to the controllability principle. To address these issues, we write $a=a^{R}$ for the realized action and take $\phi^{B}=\mathrm{E}[\phi]$ as the budgeted value of $\phi$, as suggested in the literature (Booth and Willett 1997):

$$
S=s_{0}+\mathrm{E}[\delta] a^{R}+\frac{\operatorname{Cov}[\delta, \phi]}{\operatorname{Var}[\phi]}\left(P^{R}-\phi^{B} a^{R}\right) .
$$

Thus, the agent is held responsible for the variance due to deviations of $\phi$. The controllability principle, in contrast, would demand responsibility for the measures that the agent can control (cf. Merchant and Van der Stede 2003, p. 30). On first glance, this is primarily the variance due to deviation $a^{R}-a^{B}$ of effort, which can be included

\footnotetext{
${ }^{6}$ More generally, we could consider a noisy measure $A=a+\epsilon$ of the agent's input. Owing to the agent's risk-neutrality, however, this would not affect the results of the paper.

${ }^{7}$ See Appendix B.1.
} 
in the compensation scheme by expanding the first variable part:

$$
\begin{aligned}
S & =s_{0}+\mathrm{E}[\delta] a^{R}+\left(\mathrm{E}[\delta] a^{B}-\mathrm{E}[\delta] a^{B}\right)+\frac{\operatorname{Cov}[\delta, \phi]}{\operatorname{Var}[\phi]}\left(P^{R}-\phi^{B} a^{R}\right) \\
& =\underbrace{\left(s_{0}+\mathrm{E}[\delta] a^{B}\right)}_{\tilde{s}_{0}}+\underbrace{\frac{\mathrm{E}[\delta]}{\mathrm{E}[\phi]}}_{\tilde{s}_{1}} \underbrace{\left.\left(\phi^{B} a^{R}-\phi^{B} a^{B}\right)\right)}_{\Delta^{a} P}+\underbrace{\frac{\operatorname{Cov}[\delta, \phi]}{\operatorname{Var}[\phi]}}_{\tilde{s}_{2}} \underbrace{\left(P^{R}-\phi^{B} a^{R}\right)}_{\Delta^{\phi} P} .
\end{aligned}
$$

In examining (8), we see that the agent is held responsible for both special variances, $\Delta^{a} P$ and $\Delta^{\phi} P$. Does this contradict the controllability principle? The agent obviously controls $\Delta^{a} P$ because it is the variance assigned to the deviation of $a^{R}$ and $a^{B}$. However, he also controls $\Delta^{\phi} P$, since it is computed based on the realized effort level. The first piece rate, $\tilde{s}_{1}$, motivates the agent to choose the optimal average level of effort, whereas the second part ensures optimization of his effort profile. Focusing on the effort profile highlights the importance of the refined controllability principle, which has already been emphasized in Section 2: since the principal wants the agent to care about his pre-decision information $\phi$, it is necessary to incorporate the second variance into the compensation contract. By variance decomposition, the desired effect can be delineated from the basic incentive, which was impossible under the initial contract based on $P$. This clarifies the particular value of the variance analysis: by decomposition of variances, the principal is able to fine-tune the compensation contract, stipulating different piece rates for the two variances.

The result is also in line with the informativeness principle, but this requires a comprehensive definition of what information the principal is looking for. Of course, once $a$ has been observed, the performance measure $P$ provides no additional information with respect to the agent's action. It is nevertheless useful for contracting because the principal is not interested in implementing a certain fixed effort level, but the effort level that is optimal under the agent's private information $\phi$. Therefore, even if the 
principal observes the agent's action $a$, he is still interested in inferring whether the action chosen is the one most suitable to maximize the expected total surplus. For this purpose, information on both $a$ and $\phi$ is needed, which is provided by $a$ and $P$.

The approach described conforms to the accounting literature on variance investigation as well. In general, the variance decomposition method complies with those proposed in management accounting textbooks, for instance the price and efficiency variances in cost accounting (Horngren et al. 2006, p. 227ff.). More specifically, Kloock and Schiller (1997, p. 317) state that variances computed on a budgeted basis are capable of creating proper ex ante incentives. As mentioned above, this ex ante perspective is covered by $\Delta^{a} P$. From an ex post perspective, realized amounts are considered advantageous, since they provide relevant information for planning purposes. This is fulfilled by the second variance. However, while the conventional argument refers to future planning periods in this respect, planning in the present model concerns the agent's action in the current period. The realized effort is used to quantify the (expected) benefits arising from deviations in $\phi$, motivating him to choose the right action.

Once we have shown that variance analysis procedures represent a proper instrument to implement the second-best solution in the present model, we can turn to the question of whether the inclusion of input information resolves the issue raised in Proposition 1, namely that the value of an informed agent may be negative. For this purpose, consider the agent's action $a^{V I}=s_{1}+s_{2} \phi$ resulting from the contract (5) with variance investigation. Obviously, the optimal action $a^{N I}=\mathrm{E}[\delta]$ of an uninformed agent is readily obtained by choosing $s_{1}=\mathrm{E}[\delta]$ and $s_{2}=0$, and the ambiguity of Proposition 1 disappears.

Proposition 2 In the risk-neutral agency setting with observable effort, the value of information to the agency is non-negative. 
Proof Obvious from the above considerations.

My next goal is to derive the conditions under which optimal alignment is achieved. For this purpose, compare the action

$$
a^{*}(\phi)=\mathrm{E}[\delta]+\frac{\operatorname{Cov}[\delta, \phi]}{\operatorname{Var}[\phi]}(\phi-\mathrm{E}[\phi])
$$

resulting from the second-best contract (7) to the first-best action $a^{F B}=\mathrm{E}[\delta \mid \phi]$, as given in Section 2. Obviously, the latter can be implemented by a simple linear contract of the form (1) (without observation of $a$ ) only if the expected value of $\delta$ is proportionate to $\phi$. With observation of $a$, there is an additional degree of freedom in the compensation scheme. Scheme (7) will align the interests of the agent and principal if (9) is the conditional expectation of $\delta$, given $\phi$.

Proposition 3 In the risk-neutral agency setting with observable effort, a first-best solution can be obtained by a linear contract if the conditional expectation of the agent's marginal product $\delta$ is a linear function of the sensitivity $\phi$ to his performance measure.

Proof If the expected value of $\delta$, given $\phi$, is a linear function of $\phi$, there exist $\lambda_{1}, \lambda_{2} \in \mathbb{R}$ such that $\mathrm{E}[\delta \mid \phi]=\lambda_{1}+\lambda_{2} \phi$ for all realizations of $\phi$. Using a linear contract of the form (7), the agent chooses $a(\phi)=s_{1} \mathrm{E}[\phi]+s_{2}(\phi-\mathrm{E}[\phi])$. Thus, setting $s_{2}=\lambda_{2}$ and $s_{1}=\lambda_{2}+\lambda_{1} / \mathrm{E}[\phi]$ yields the first-best action $a^{* *}=\mathrm{E}[\delta \mid \phi]$.

If the conditional expectation of $\delta$ is a linear function of $\phi$, can it be replicated by a linear contract. ${ }^{8}$ A sufficient (but not necessary) condition for the linearity requirement

\footnotetext{
${ }^{8} \mathrm{We}$ could look for distributions that meet this condition and allow for the first-best solution. This is obviously the case for all distributions meeting the linear conditional expectation. A well-known example is the class of elliptical distributions, of which the normal distribution is a special case. A larger class is the Pearson family (see Wei et al. 1999 for details). Beyond this, discrete distributions that fulfil the requirement can also be constructed.
} 
is perfect correlation of $\delta$ and $\phi$, in which case $\delta$ itself is a linear function of $\phi$ by definition. Moreover, under perfect correlation the first-best outcome is identical to the full-information outcome, where the principal has perfect knowledge of $\delta$.

Corrollary 1 If $\delta$ and $\phi$ are perfectly correlated in the risk-neutral agency setting with observable effort, the full-information solution can be obtained by a linear contract.

Proof Under perfect correlation, a linear relation $\delta=\lambda_{1}+\lambda_{2} \phi$ holds for some $\lambda_{1}, \lambda_{2} \in \mathbb{R}$ and all realizations of $\delta$ and $\phi$. Setting $s_{2}=\lambda_{2}$ and $s_{1}=\lambda_{2}+\lambda_{1} \mathrm{E}[\phi]$ induces $a=\delta$, which yields the full-information solution.

Corollary 1 is a special case of Proposition 3. Under perfect correlation, $\delta$ can be inferred unambiguously from $\phi$, and symmetric information is equivalent to full information. Thus, it seems obvious that, contrary to the example of Section 2, the correlation of $\delta$ and $\phi$ has a positive impact on the principal's benefit, at least if the conditions of Proposition 1 are fulfilled. To analyze this in general, we denote the correlation of $\delta$ and $\phi$ by $\rho$. The agent's action (9) under the two-piece-rate contract (7) can then be written as

$$
a^{V I}=\mathrm{E}[\delta]+\rho \sqrt{\frac{\operatorname{Var}[\delta]}{\operatorname{Var}[\phi]}}(\phi-\mathrm{E}[\phi]) .
$$

By substitution of this term in the principal's objective function, the expected total surplus becomes ${ }^{9}$

$$
\mathrm{E}[V-C]^{V I}=\frac{1}{2}\left(\mathrm{E}[\delta]^{2}+\rho^{2} \operatorname{Var}[\delta]\right) .
$$

A comparison of (10) and (3) reveals that, contrary to the situation without observable effort, the performance measure now affects the agency's surplus only by the correla-

\footnotetext{
${ }^{9}$ See Appendix B.2.
} 
tion of $\delta$ and $\phi$. The latter therefore perfectly indicates the quality of a performance measure for a linear contract.

Proposition 4 In the risk-neutral agency setting with observable effort, the agency's surplus is increasing in the correlation between the agent's marginal product $\delta$ and the sensitivity $\phi$ of his performance measure.

Proof Obvious from Eq. (10).

Proposition 4 condenses the insight that in the risk-neutral agency with distorted performance measurement, dealing with the agent's private information is merely a matter of inferring his marginal product from the sensitivity of the performance measure applied. Observing the agent's effort enables the principal to use a linear regression, where the correlation coefficient indicates the quality of the inference.

\section{Participative budgeting}

So far I have assumed that the agent's private information cannot be communicated to the principal because of timing effects or limited information channels. Without this restriction, an obvious device to improve effort allocation is the agent's participation in budgeting. In economic terms, this corresponds to offering a menu of contracts to the agent. In this respect, most of the literature has focussed on the tradeoff of information rents and inefficiencies of allocation (cf. Antle and Eppen 1988 and Antle and Fellingham 1990 as the most prominent examples). Since in the present model the agent is risk-neutral and acquires private information only after he has signed the contract, however, implementation is without an additional cost because any information rent can be extracted by an upfront payment. The problem therefore reduces to the question of whether a certain action profile is implementable. 
A well-known result in this respect is that under optimal contracting, using a menu of contracts is of no value compared to a single contract if the agent's action and his private information unambiguously determine the outcome, except for additive noise (Melumad and Reichelstein 1989; Caillaud et al. 1992). In the present model, however, contract design is restricted in two respects. First, I have confined myself to the analysis of linear contracts, which is generally not be capable of mimicking the allocation of a menu of contracts. ${ }^{10}$ Second, in contrast to the above results, the contract can only be written on the distorted performance measure $P$, which further restricts the design of incentives. Hence, although the agent's performance is determined by his action $a$ and private information $\phi$, and is only perturbed by white noise $\epsilon$, menus of contracts may help to make further use of his knowledge of $\phi$.

The extent to which the agent's private information can be exploited depends on what the principal can observe. If $a$ is observable, the first-best solution can easily be achieved by prescribing the first-best effort $a(\hat{\phi})=\mathrm{E}[\delta \mid \hat{\phi}]$ for any announced level $\hat{\phi}$ of $\phi$, thereby compensating the agent for the resulting cost of effort. This eliminates the moral hazard problem, and budget participation becomes merely a matter of eliciting the agent's private information, which can be achieved by a mechanism according to Osband and Reichelstein (1985), in which deviations of budgeted and realized performance are punished by a convex incentive scheme ${ }^{11}$

$$
S(\hat{P}, P)=l(\hat{P})+l^{\prime}(\hat{P})[P-\hat{P}]
$$

where $\hat{P}=\hat{\phi} a(\hat{\phi})$ denotes the budgeted performance, and $l(\cdot)$ is an arbitrary convex

\footnotetext{
${ }^{10}$ Such a contract would be the upper envelope of contingent contracts, which will hardly be linear.

${ }^{11}$ Another well-known mechanism is that proposed by Weitzman (1976). Unlike the scheme of Osband and Reichelstein, however, the Weitzman scheme would reveal a quantile of the distribution of $P$ instead of its expectation.
} 
function. ${ }^{12}$ Such penalties again introduce the structure of variance analysis into the contract, but as a means of disciplining the agent in participative budgeting rather than inferring the agent's private information, as in the framework without communication.

If $a$ cannot be contracted on, the problems of moral hazard and private information cannot be separated, and a more subtle contract has to be used. Again, the agent will not earn an informational rent because he obtains private information only after contracting. However, the first-best solution can only be achieved if certain regularities are met. To examine this subject, I confine myself to the analysis of menus of linear contracts, following my previous analysis of cases without communication. For a linear contract $S(P, \hat{\phi})=s_{0}(\hat{\phi})+s_{1}(\hat{\phi}) P$, conditional to the announced value $\hat{\phi}$ of the agent's private information, the agent will choose an effort level $a(\hat{\phi})=s_{1}(\hat{\phi}) \phi$, and his expected utility under this action choice is given by

$$
W(\hat{\phi}, \phi) \equiv \mathrm{E}\left[U\left(s_{0}(\hat{\phi}), s_{1}(\hat{\phi}), \phi\right)\right]=s_{0}(\hat{\phi})+\frac{s_{1}(\hat{\phi})^{2} \phi^{2}}{2}
$$

For this indirect utility, the problem can be analyzed using the standard model of mechanism design (Salanie 1997, p. 26ff.; Fudenberg and Tirole 1991, p. 257ff.). A familiar result is that if the marginal rate of substitution between the allocation (in this case $\left.s_{1}(\hat{\phi})\right)$ and transfer $\left(s_{0}(\hat{\phi})\right)$ has a constant sign (the so-called single-crossing or sorting condition) and is bounded for all transfers, any non-decreasing/non-increasing allocation is implementable-depending on the sign of the sorting condition (Guesnerie and Laffont 1984, Theorem 2). Under an increasing allocation $s_{1}(\cdot)$, for example, the agent must be willing to pay more (i.e. to accept higher cuts in his base salary) for an increase in his share $s_{1}$ if he observes higher values of $\phi$. Then, different types

\footnotetext{
${ }^{12}$ Since $P$ is distorted by white noise, the agent in this case has to be compensated for the expected punishment under truthful reporting. Due to the agent's risk-neutrality, such sanctions are free of cost to the principal.
} 
of agents can be separated by offering larger variable payments for higher-sensitivity products and making the agent pay for this privilege. ${ }^{13}$

Based on this rationale, it can be studied under which conditions the first-best allocation can be induced, and what type of contract results. The first issue reduces to the question of whether the regularity conditions are met for the first-best effort level $a^{F B}=\mathrm{E}[\delta \mid \phi]$.

Proposition 5 In the risk-neutral agency setting without observable effort, the firstbest action can be implemented by a menu of linear contracts if $E[\delta \mid \phi] / \phi$ is an increasing function of $\phi$.

\section{Proof See Appendix A.}

Proposition 5 proves that the first-best action can be induced if the respective share parameter $s_{1}^{0}$ is an increasing function of the agent's information $\phi$. That is, not only should a higher effort be optimal for higher levels of $\phi$, but it also needs to be implemented by a higher share of $P .{ }^{14}$ A decreasing first-best allocation is not implementable because the agent would always pretend to be of type $\underline{\phi}$, irrespective of his observation.

Separation of agents also does not happen if $\mathrm{E}[\delta \mid \phi] / \phi$ is constant, in which case the same contract is offered for all announced values of $\phi$. As is easily seen, this refers to a situation where $\mathrm{E}[\delta \mid \phi]$ is proportionate to $\phi$, and the first-best action can be induced by a single linear contract (see Section 2). If $s_{1}^{0}$ is strictly increasing, the firstbest allocation can be induced by a revelation mechanism that discriminates between

\footnotetext{
${ }^{13}$ For a decreasing allocation, this logic has to be reversed.

${ }^{14}$ This limitation arises because the sorting condition is always fulfilled in its positive form, as described above.
} 
types. The corresponding menu of linear contracts is given by ${ }^{15}$

$$
\begin{aligned}
S^{0}(\hat{\phi}) & =s_{0}^{0}(\hat{\phi})+s_{1}^{0}(\hat{\phi}) P \\
& =W(\underline{\phi}, \underline{\phi})+\int_{\underline{\phi}}^{\hat{\phi}} \frac{\mathrm{E}[\delta \mid \varphi]^{2}}{\varphi} d \varphi+\frac{\mathrm{E}[\delta \mid \hat{\phi}]^{2}}{2}+\frac{\mathrm{E}[\delta \mid \hat{\phi}]}{\hat{\phi}}(P-\mathrm{E}[\delta \mid \hat{\phi}] \hat{\phi}) .
\end{aligned}
$$

Again, the last term exhibits a variance structure, which can further be clarified by fixing budgets $\phi^{B}=\hat{\phi}$ and $a^{B}=\mathrm{E}[\delta \mid \hat{\phi}]$ :

$$
S^{0}\left(\phi^{B}\right)=W(\underline{\phi}, \underline{\phi})+\underbrace{\int_{\phi}^{\phi^{B}} \frac{\mathrm{E}[\delta \mid \varphi]^{2}}{\varphi} d \varphi}_{\text {revelation term }}+\underbrace{\frac{\left(a^{B}\right)^{2}}{2}}_{\text {effort compensation }}+\underbrace{s_{1}\left(\phi^{B}\right)\left[P-a^{B} \phi^{B}\right]}_{\text {variance analysis term }}
$$

The budget $\phi^{B}$ is set by the agent, yielding the budgeted effort $a^{B}=\mathrm{E}\left[\delta \mid \phi^{B}\right]$ in a prespecified way. Under truthful reporting, the variance term has an expected value of zero, and the agent will be compensated for his disutility of effort. To achieve truthtelling, higher values of $\phi$ have to yield higher utilities, which is guaranteed by the revelation term on the left. Finally, the base salary $W(\underline{\phi}, \underline{\phi})$ for the least profitable type $\underline{\phi}$ is fixed to provide the agent's reservation utility. The structure of the compensation contract shows that, in contrast to the setting with observable effort and no communication, the agent is made responsible for the whole deviation $P-a^{B} \phi^{B}=P-P^{B}$ of realized and budgeted performance, without any distinction in the magnitude of incentives. Different rates for different special variances are not necessary under participative budgeting because the agent's private information is revealed by his choice among the contracts offered. The average level of effort, as well as its distinction according to the marginal product, is controlled in a single step. Once the agent has committed to a certain budget, the total variance suffices to ensure that he will not differ from the corresponding action. This is well in line with the controllability principle: since

\footnotetext{
${ }^{15}$ See Appendix B.3.
} 
the agent observes $\phi$ and fixes the budget $P^{B}=\phi^{B} a^{B}$ by announcing $\phi^{B}$, he fully controls the deviation $P-P^{B}$, except for the additive noise. Since the latter cannot be factored out, rewarding $P-P^{B}$ is the best the principal can do if he does not observe $a$.

By comparison of (12) to the incentive scheme (11) with observable effort, the benefits from additional input information become obvious: although both schemes exhibit a similar structure, $S^{0}$ is limited because, contrary to (11), in (12) it does not suffice to punish deviation by an arbitrary increasing function $l^{\prime}(\cdot)$. The share parameter also has to induce the first-best action, which under observable effort is achieved by a forcing contract. With unobservable effort, the contract has to serve two purposes, which—as Proposition 5 shows_ can only be brought in line under certain conditions. Thus, at least with regard to the first-best allocation, the observation of $a$ will be of value whenever the requirement of Proposition 5 is not fulfilled.

Finally, to return to the starting point of this section, we see that, in contrast to the classical models on communication in agencies (Melumad and Reichelstein 1989; Caillaud and Guesnerie 1992), budget participation may well be of value in the present model, despite its simple informational assumptions: with observable effort, communication is of value whenever the requirements of Proposition 3 are not met. Without this input information, Proposition 5 shows that communication is of value if the firstbest action is implemented in a separating equilibrium. This highlights the fact that performance measure distortion substantially limits the use of participative budgeting: if budgets refer to $V$ instead of $P$, implementing the first-best solution would of course not be an issue. Thus, the restriction to linear contracts alone would not warrant the use of participative budgeting, which is only of value under distorted performance measurement. 


\section{Conclusion}

This paper has analyzed the role of variance investigation procedures in mitigating the problem of effort misallocation in an agency setting with distorted performance measurement. It was shown that variance analysis improves the optimal linear contract in most cases for which the performance measure is not in line with the principal's objective. By application of variance investigation, the quality of a particular performance measure for a privately informed agent can be quantified using the correlation of its marginal product and that of the principal's objective. Special variances have a natural interpretation in this setting: they quantify the expected deviation from the principal's objective, given a performance measure deviation. Using deviations instead of total amounts, the effects of measured performance can be translated into value effects. Thus, the objectives of the principal and agent can be aligned via compensation.

If the agent can communicate his private information, participative budgeting changes the role of variance analysis. While under top-down budgeting the agent is held responsible for the two variances to different extents, under participation he bears responsibility for the overall deviation of realized and budgeted performance. Such complete stewardship, however, is feasible only under certain conditions. Otherwise, additional information on the agent's input may be used to achieve complete alignment.

The results of this paper can be related to the recent discussion of strategic performance measurement. My starting point was the exclusive use of financial accounting measures, which have been considered incongruent with the long-term objectives of the firm. In this paper, traditional accounting practices, namely variance analysis and participative budgeting, have been proven capable of alleviating this incongruity. It was found that variance analysis particularly helps in situations in which the agent's marginal products are strongly correlated with the sensitivities of the (retrospective) 
accounting measures.

Such correlation occurs in situations in which a manager's impact on the firm's long-term objectives can be separated into an undirected effect related to the absolute level of his activities and a directed effect proportional to his short-term objectives (including profit). Taking into account that current profit is an inherent part of the firm's overall financial objective, such situations may best be described by a stable environment, in which current opportunities $(\phi)$ represent a good predictor of the long-term prospects of success $(\delta)$, or a business with little strategic focus, in which the firm's long-term objectives mainly depend on the absolute level of the manager's current activities, but there is no concrete plan describing how this effort should best be allocated.

None of these scenarios fully complies with the competitive environment for which strategic measurement systems are proposed. Therefore, the results of the paper do not overcome the necessity for additional (non-financial) measures to align the objectives of organizational members with the firm's strategy. They rather provide evidence for situations in which their application appears to be less essential.

\section{A Proof of Proposition 5}

Let $s_{1}(\phi)$ be the allocation to be implemented. A necessary and sufficient condition for $s_{1}$ to be implementable is

$$
\left.\frac{\partial^{2}}{\partial s_{1} \partial \phi}\left(\frac{s_{1}(\hat{\phi})^{2} \phi^{2}}{2}\right) \frac{d s_{1}}{d \phi}(\phi)\right|_{\hat{\phi}=\phi}=\left(2 s_{1}(\phi) \phi\right) \frac{d s_{1}}{d \hat{\phi}}(\phi) \geq 0
$$

meaning that the sensitivity product $\phi$ affects the agent's marginal utility from an increase in $s_{1}$ in a systematic way (Salanie 1997, p. 30). The sorting condition requires that the cross-derivative in (A.1) is positive $\left(S C^{+}\right)$or negative $\left(S C^{-}\right)$for all $\phi . \mathrm{Un}$ der the additional assumption that the cross-derivative is smaller than $K_{0}+K_{1}\left|s_{0}\right|$ for 
some $K_{0}, K_{1} \in \mathbb{R}$ and all $\phi$, any non-decreasing (for $S C^{+}$) or non-increasing (for $S C^{-}$) allocation is implementable (Guesnerie and Laffont 1984, Theorem 2).

The second regularity condition is met because the supports of both $\phi$ and $\delta$ are bounded. Therefore, substitution of the first-best share parameter $s_{1}^{0}(\hat{\phi})=\mathrm{E}[\delta \mid \hat{\phi}] / \hat{\phi}$ in (A.1) yields

$$
\left(2 s_{1}^{0}(\phi) \phi\right) \frac{d s_{1}^{0}}{d \hat{\phi}}(\phi)=2 \mathrm{E}[\delta \mid \phi] \frac{d}{d \phi}\left(\frac{\mathrm{E}[\delta \mid \phi]}{\phi}\right) \geq 0
$$

as a necessary condition for the first-best solution to be implementable. Since by assumption the marginal product $\delta$ of the agent's action is positive, the same holds for its expectation $\mathrm{E}[\delta \mid \phi]$. Thus, the sorting condition $C S^{+}$is always met, and condition (A.2) reduces to

$$
\frac{d}{d \phi}\left(\frac{\mathrm{E}[\delta \mid \phi]}{\phi}\right) \geq 0
$$

where the desired allocation is non-decreasing in the agent's information $\phi$. Due to the sorting condition $C S^{+}$, condition (A.3) is also sufficient for implementation (Salanie 1997, p. 31).

\section{B Further computations}

\section{B.1 Contract parameters (6)}

Based on $P$ and $a$, a linear contract is of the form $S=s_{0}+s_{1} a+s_{2} P$, resulting in an action choice $a(\phi)=s_{1}+s_{2} \phi$. Substituting this into the principal's objective of maximizing total surplus

$$
\mathrm{E}[V-S]=\mathrm{E}\left[\delta a(\phi)-\frac{a(\phi)^{2}}{2}\right]
$$


yields the optimization problem

$$
\max _{s_{1}, s_{2}} \mathrm{E}[V-S]=s_{1} \mathrm{E}[\delta]+s_{2} \mathrm{E}[\delta \phi]-\frac{s_{1}^{2}}{2}-s_{1} s_{2} \mathrm{E}[\phi]-\frac{s_{2}^{2}}{2} \mathrm{E}\left[\phi^{2}\right]
$$

From the first-order conditions

$$
\frac{\partial \mathrm{E}[V-S]}{\partial s_{1}}=\mathrm{E}[\delta]-s_{1}-s_{2} \mathrm{E}[\phi]=0
$$

and

$$
\frac{\partial \mathrm{E}[V-S]}{\partial s_{2}}=\mathrm{E}[\delta \phi]-s_{1} \mathrm{E}[\phi]-s_{2} \mathrm{E}\left[\phi^{2}\right]=0
$$

I derive

$$
s_{1}=\mathrm{E}[\delta]-s_{2} \mathrm{E}[\phi] \quad \text { and } \quad s_{2}=\frac{\mathrm{E}[\delta \phi]-\mathrm{E}[\delta] \mathrm{E}[\phi]}{\mathrm{E}\left[\phi^{2}\right]-\mathrm{E}[\phi]^{2}}=\frac{\operatorname{Cov}[\delta \phi]}{\operatorname{Var}[\phi]}
$$

for the optimal values of $s_{1}$ and $s_{2}$, respectively, which coincides with (7).

\section{B.2 Expected surplus from a contract with two piece rates}

The agent's incentive constraint yields

$$
a^{*}=s_{1}+s_{2} \phi=\mathrm{E}[\delta]+s_{2}(\phi-\mathrm{E}[\phi])
$$


where I use the fact that $s_{1}=\mathrm{E}[\delta]-s_{2} \mathrm{E}[\phi]$. Substituting this into the gross profit yields

$$
\begin{aligned}
V-C & =\delta a^{*}-\frac{\left(a^{*}\right)^{2}}{2} \\
& =\delta\left[s_{1}+s_{2} \phi\right]+\frac{\left[s_{1}+s_{2} \phi\right]^{2}}{2} \\
& =\delta\left[\mathrm{E}[\delta]+s_{2}(\phi-\mathrm{E}[\phi])\right]-\frac{\left[\mathrm{E}[\delta]+s_{2}(\phi-\mathrm{E}[\phi])\right]^{2}}{2} .
\end{aligned}
$$

Computation of expectations gives

$$
\begin{aligned}
\mathrm{E}[V-C] & =\mathrm{E}[\delta]^{2}+s_{2} \mathrm{E}[\delta \phi-\delta \mathrm{E}[\phi]] \\
& -\frac{1}{2}\left(\mathrm{E}[\delta]^{2}+2 s_{2} \mathrm{E}[\delta] \mathrm{E}[\phi-\mathrm{E}[\phi]]+s_{2}^{2} \mathrm{E}[\phi-\mathrm{E}[\phi]]^{2}\right) \\
& =\frac{1}{2} \mathrm{E}[\delta]^{2}+s_{2} \operatorname{Cov}[\delta, \phi]-\frac{1}{2} s_{2}^{2} \operatorname{Var}[\phi]
\end{aligned}
$$

Substitution of $s_{2}=\frac{\operatorname{Cov}[\delta, \phi]}{\operatorname{Var}[\phi]}$ yields

$$
\begin{aligned}
\mathrm{E}[V-C] & =\frac{1}{2} \mathrm{E}[\delta]^{2}+\frac{\operatorname{Cov}[\delta, \phi]}{\operatorname{Var}[\phi]} \operatorname{Cov}[\delta, \phi]-\frac{1}{2} \frac{\operatorname{Cov}[\delta, \phi]^{2}}{\operatorname{Var}[\phi]^{2}} \operatorname{Var}[\phi] \\
& =\frac{1}{2}\left(\mathrm{E}[\delta]^{2}+\frac{\operatorname{Cov}[\delta, \phi]^{2}}{\operatorname{Var}[\phi]}\right)=\frac{1}{2}\left(\mathrm{E}[\delta]^{2}+\rho^{2} \operatorname{Var}[\delta]\right)
\end{aligned}
$$

\section{B.3 Derivation of the revelation mechanism}

The agent's marginal utility under truth-telling and $s_{1}^{0}$ is given by ${ }^{16}$

$$
\frac{d}{d \phi} W(\phi, \phi)=\frac{\partial}{\partial \phi} W(\hat{\phi}, \phi)=\left(s_{1}^{0}(\phi)\right)^{2} \phi=\frac{\mathrm{E}[\delta \mid \phi]^{2}}{\phi} .
$$

\footnotetext{
${ }^{16}$ For the following, cf. Salanie (1997) p. 33f.
} 
From this, I can derive the utility

$$
W(\phi, \phi)=W(\underline{\phi}, \underline{\phi})+\int_{\underline{\phi}}^{\phi} \frac{\mathrm{E}[\delta \mid \varphi]^{2}}{\varphi} d \varphi
$$

for an agent of type $\phi$, which yields the required fixed payment

$$
\begin{aligned}
s_{0}^{0}(\phi) & =W(\phi, \phi)-\frac{s_{1}^{0}(\phi)^{2} \phi^{2}}{2} \\
& =W(\underline{\phi}, \underline{\phi})+\int_{\underline{\phi}}^{\phi} \frac{\mathrm{E}[\delta \mid \varphi]^{2}}{\varphi} d \varphi-\frac{\mathrm{E}[\delta \mid \phi]^{2}}{2} .
\end{aligned}
$$

Finally, $W(\underline{\phi}, \underline{\phi})$ is chosen to meet the agent's participation constraint. The resulting total compensation $S(\phi)=s_{0}^{0}(\phi)+s_{1}^{0}(\phi)$ is

$$
\begin{aligned}
S^{0}(\phi) & =s_{0}^{0}(\phi)+s_{1}^{0}(\phi) \\
& =W(\underline{\phi}, \underline{\phi})+\int_{\underline{\phi}}^{\phi} \frac{\mathrm{E}[\delta \mid \varphi]^{2}}{\varphi} d \varphi-\frac{\mathrm{E}[\delta \mid \phi]^{2}}{2}+\frac{\mathrm{E}[\delta \mid \phi]}{\phi} P \\
& =W(\underline{\phi}, \underline{\phi})+\int_{\underline{\phi}}^{\phi} \frac{\mathrm{E}[\delta \mid \varphi]^{2}}{\varphi} d \varphi+\frac{\mathrm{E}[\delta \mid \phi]^{2}}{2}+\frac{\mathrm{E}[\delta \mid \phi]}{\phi}(P-\mathrm{E}[\delta \mid \phi] \phi) .
\end{aligned}
$$

\section{References}

[1] Antle, R., Eppen, G.D., 1985. Capital rationing and organizational slack in capital budgeting. Manage. Sci. 31, 163-174.

[2] Antle, R., Fellingham, J., 1990. Resource rationing and organizational slack in a two-period model. J. Acc. Res. 28(1), 1-24.

[3] Baiman, S., Demski, J.S., 1980a. Variance analysis procedures as motivational devices. Manage. Sci. 26(8), 840-848.

[4] Baiman, S., Demski, J.S., 1980b. Economically optimal performance evaluation and control system. J. Acc. Res. 18, 184-220. 
[5] Baker, G.P., 1992. Incentive contracts and performance measurement. J. Polit. Econ. 100(3), 598-614.

[6] Banker, R.D., Datar, S.M., 1989. Sensitivity, precision, and linear aggregation of signals for performance evaluation. J. Acc. Res. 27(1), 21-39.

[7] Booth, J.G., Willett, R., 1997. The statistical analysis of standard-cost deviations. IMA J. Manage. Math. 8, 167-179.

[8] Caillaud, B., Guesnerie, R., Rey, P., 1992. Noisy observation in adverse selection models. Rev. Econ. Stud. 59, 595-615.

[9] Christensen, J., 1981. Communication in agencies. Bell J. Econ. 12(2), 661-674.

[10] Darrough, M.N., 1988. Variance analysis: a unifying cost function approach. Contemp. Acc. Res. 5(1), 199-221.

[11] Demski, J.S., 1980. Information Analysis. Addison-Wesley, Reading, MA.

[12] Feltham, G.A., Xie, J., 1994. Performance measure congruity and diversity in multi-task principal/agent relations. Acc. Rev. 69(3), 429-453.

[13] Fudenberg, D., Tirole, J., 1991, Game Theory. MIT Press, Cambridge, MA.

[14] Guesnerie, R., Laffont, J.-J., 1984. A complete solution to a class of principalagent problems with an application to the control of a self-managed firm. J. Public Econ. 25, 329-369.

[15] Harris, M., Raviv, A., 1979. Optimal incentive contracts with imperfect information. J. Econ. Theory 20, 231-259.

[16] Holmström, B., 1979. Moral hazard and observability. Bell J. Econ. 10, 74-91. 
[17] Holmström, B., Milgrom, P., 1991. Multitask principal-agent analyses: incentive contracts, asset ownership, and job design. J. Law Econ. Organ. 7, 24-52.

[18] Horngren, C.T., Datar, S.M., Foster, G., 2006. Cost Accounting: A Managerial Emphasis. 12th ed., Prentice-Hall, Upper Saddle River, NJ.

[19] Kloock, J., Schiller, U., 1997. Marginal costing: cost budgeting and cost variance analysis. Manage. Acc. Res. 8(3), 299-324.

[20] Melumad, N.D., Reichelstein, S., 1989. Value of communication in agencies. J. Econ. Theory 47, 334-368.

[21] Merchant, K.A., Van der Stede, W.A., 2003. Management Control Systems: Performance Measurement, Evaluation, and Incentives. Pearson Education, Harlow, UK.

[22] Osband, K., Reichelstein, S., 1985. Information eliciting compensation schemes. J. Public Econ. 27, 107-16.

[23] Salanie, B., 1997, The Economics of Contracts: A Primer. MIT Press, Cambridge, MA.

[24] Wei, K.C.J., Lee, C.F., Lee, A.C., 1999. Linear conditional expectation, return distributions, and capital asset pricing theories. J. Finan. Res. 22(4), 471-487.

[25] Weitzman, M.L., 1976. The new Soviet incentive model. Bell J. Econ. 7, 251-57.

[26] Young, R. A., 1986. A note on "Economically optimal performance evaluation and control systems": The optimality of two-tailed investigations. J. Acc. Res. 24(1), 231-240. 